\title{
Um novo clorofilômetro para estimar os teores de clorofila em folhas do capim Tifton 85
}

\author{
A new chlorophyll meter to estimate chlorophyll contents in leaves of Tifton 85 bermudagrass
}

\author{
Élio Barbieri Junior ${ }^{{ }^{*}}$ Roberto Oscar Pereyra Rossiello ${ }^{\mathrm{I}}$ Rosani Valéria Marcelina Matoso Silva \\ Roberta Cristiane Ribeiro ${ }^{\text {II }}$ Mirton José Frota Morenz ${ }^{\text {II }}$
}

\begin{abstract}
O propósito deste trabalho foi verificar o desempenho de um novo clorofilometro portátil, em parcelas experimentais vegetadas com o capim-Tifton 85 (Cynodon spp.). Para tal, foi utilizado um experimento de curta duração, em que três doses de $N\left(0,75\right.$ e $150 \mathrm{~kg}$ de $\left.\mathrm{N} \mathrm{ha} \mathrm{a}^{-1}\right)$ foram combinadas com quatro datas de amostragem $(8,16,24$ e 28 dias após um corte de uniformização). As análises foram feitas em lâminas da folha mais nova totalmente expandida. Os teores das clorofilas $a, b e$ $a+b$ foram quantificados espectrofotometricamente após a extração com acetona $80 \%$, enquanto que o clorofilômetro forneceu os correspondentes valores de Índice de Clorofila Falker (ICF). As leituras do aparelho foram sensíveis tanto às doses de $N$ quanto às datas de amostragem. Foram obtidas correlações positivas entre ambos os métodos de quantificação (clorofila a: $r=0,646 ; b: r=0,797 ;$ e total: $r=0,721$ ), que evidenciaram $a$ utilidade desse novo clorofilometro na determinação indireta dos teores de clorofila foliar no capim-Tifton 85.
\end{abstract}

Palavras-chave: Cynodon spp., clorofila total, adubação nitrogenada.

\section{ABSTRACT}

The purpose of this research was to evaluate the performance of a new portable chlorophyll meter in the indirect determination of chlorophyll contents in Tifton 85 bermudagrass leaves. In a field trial of short duration, three $N$ doses $(0,75$ and $\left.150 \mathrm{~kg} \mathrm{ha}^{-1}\right)$ were combined with four sampling dates $(8$, 16, 24 e 28 days after a cut for sward uniformity) in completely randomized blocks, with treatments arranged in a split-plot design. Analyses were performed in the youngest fully expanded leaf blade. Contents of chlorophylls $a, b$ and total were quantified spectrophotometrically, after extraction with acetone
$80 \%$, while chlorophyll meter readings, expressed as Falker Chlorophyll Index (FCI) units, provided the same information. Chlorophyll meter readings were sensitive to both $N$ rates and sampling dates. It were obtained significant positive correlations between both methods (chlorophyll a: $r=0.646$, chlorophyll $b$ : $r=0.797$ and for total chlorophyll: $r=0.721$ ). It was concluded that this new chlorophyll meter constitutes an appropriate tool for the indirect determination of foliar chlorophyll contents in Tifton 85 pastures.

Key words: Cynodon spp., nitrogen fertilization, total chlorophyll.

Os clorofilometros são instrumentos que aferem, de forma indireta e não destrutiva, os teores de clorofila com base nas propriedades óticas das folhas (ARGENTAet al., 2001a). O primeiro e mais difundidoé o SPAD (Soil Plant Analysis Development, Konica Minolta, Japão), o qual possui diodos que emitem luz em comprimentos de onda $(\lambda)$ de $650 \mathrm{~nm}$ (vermelho) e a $940 \mathrm{~nm}$ (infravermelho). A emissão em $\lambda=650 \mathrm{~nm}$ situa-se próxima dos comprimentos de ondas associados à atividade da clorofila, enquanto que a emissão em $\lambda=940 \mathrm{~nm}$ serve como referência interna para compensar diferenças na espessura ou no conteúdo hídrico da folha (MARKWELLet al., 1995). Outro equipamento similar, chamado $N$-Tester (Yara International, Noruega), tem sido aplicado à identificação de desordens nutricionais

IPrograma de Pós-graduação em Zootecnia, Universidade Federal Rural do Rio Janeiro (UFRRJ), 23890-000, Seropédica, RJ, Brasil. E-mail: elio.barbieri@cfs.ifmt.edu.br.*Autor para correspondência.

"Instituto Federal do Mato Grosso (IFMT), Campus Confresa, Confresa, MT, Brasil.

IIIEmbrapa Gado de Leite, Juiz de Fora, MG, Brasil. 
e à determinação de níveis críticos de N-foliar (ORTUZAR-IRAGORRI et al., 2005). De mais recente lançamento, o clorofilometro chamado ClorofiLOG (Falker Automação Agrícola, Brasil), utiliza fotodiodos emissores em três comprimentos de onda (FALKER, 2008): dois emitem dentro da banda do vermelho, próximos aos picos de cada tipo de clorofila $(\lambda=635 \mathrm{e}$ $660 \mathrm{~nm}$ ) eum outrono infravermelhopróximo ( $\lambda=880 \mathrm{~nm})$. Da mesma forma que oSPAD, um sensor inferior recebe a radiação transmitida através da estrutura foliar. A partir desses dados, o aparelho fornece valores chamados Índice de Clorofila Falker (ICF) proporcionais à absorbância das clorofilas. O propósito do presente trabalho é verificar o desempenho desse novo clorofilometro, através da relação das suas leituras com teores de clorofila extraídos diretamente, visando a sua utilização no manejo da adubação nitrogenada em pastagens de Cynodon spp. cv. 'Tifton 85'.

O experimento foi realizado na Universidade Federal Rural do Rio de Janeiro, Seropédica, RJ, em área preparada especificamente para este experimento. O plantio foi realizado utilizando-se estolões enraizados provenientes de área contigua, em sulcos separados de $0,5 \mathrm{~m}$, em parcelas de $4 \times 4 \mathrm{~m}$. Previamente, foi feita uma aplicação basal de $\mathrm{P}\left(150 \mathrm{~kg} \mathrm{ha}^{-1}\right)$, no fundo do sulco, e, no pós-plantio, foi aplicada adubação potássica ( $80 \mathrm{~kg}$ de $\mathrm{K} \mathrm{ha}^{-1}$, como KCl, em cobertura). $\mathrm{O}$ experimento foi disposto segundo um desenho em blocos inteiramente casualizados, com quatro repetições e três doses de $\mathrm{N}$, de forma a induzir níveis diferenciados do nutriente no solo: $0 ; 75$ e $150 \mathrm{~kg} \mathrm{Nha}^{-1}$, na forma de ureia. As determinações experimentais foram realizadas entre 30 de novembro e 28 de dezembro de 2008, período no qual foram feitas quatro amostragens, expressas como dias após um corte de uniformização (DAC). As primeiras três datas foram prefixadas (8, 16 e 24 DAC), enquanto a última foi realizada quando os dosséis de maior crescimento atingiram valores de interceptação da radiação fotossinteticamente ativa dentro da faixa $93-95 \%$, conforme avaliado com sensor de quantum. Usou-se a primeira folha totalmente expandida (do topo para a base do perfilho), exposta à radiação solar. No terço médio da lâmina, foram feitas três leituras com o clorofilometro ClorofiLOG, modelo CFL 1030, operado de acordo com as especificações do fabricante (FALKER, 2008). Esse procedimento foi repetido em 10 folhas por parcela. Imediatamente após as leituras, retirou-se, com auxílio de um furador de papel, um disco foliar $\left(28,3 \mathrm{~mm}^{2}\right.$ folha $\left.\mathrm{a}^{-1}\right)$, totalizando uma amostra composta de $283 \mathrm{~mm}^{2}$ de tecido verde/10 discos. Esse material foi imediatamente transferido a frascos de vidro contendo 20ml de acetona 80\% (ARNON, 1949), procedendo-se à determinação espectrofotométrica das clorofilas $\mathrm{a}$; $\mathrm{b}$ e total $(\mathrm{a}+\mathrm{b})$, conforme descrito por BARBIERI JUNIOR et al. (2010). Os dados foram submetidos a análises de variância e de correlação paramétrica, utilizando-se para tal o software GraphPad Prism v. 5.0 (MOTULSKY \& CHRISTOPOULOS, 2004).

Os teores de clorofila total (CFT) extraídos com acetona $80 \%$ variaram entre 264 e $699 \mu \mathrm{mol} \mathrm{m}^{-2}$, em decorrência de efeitos significativos da dose de $\mathrm{N}$ $(\mathrm{P}<0,0001)$, DAC $(\mathrm{P}<0,0001)$ e dose $\mathrm{xAC}(\mathrm{P}=0,0002)$. Em média, a adubação nitrogenada aumentou os teores de CFT em 58\%, considerando-se a maior dose aplicada em relação ao controle $\left(585 \pm 45\right.$ vs. $\left.370 \pm 69 \mu \mathrm{mol} \mathrm{m}^{-2}\right)$. Todavia, o maior contraste entre doses aconteceu aos 24DAC, quando a diferença a favor da maior dose foi de $69 \%$ (648 $\left.\pm 44 v s .384 \pm 47 \mu \mathrm{mol} \mathrm{m}^{-2}\right)$. A variação das leituras do clorofilometro seguiu um padrão inteiramente similar, sendo afetado $(\mathrm{P}<0,0001)$ pelos fatores dose de N, DAC e pela sua interação. Excetuando-se as leituras correspondentes às doses de 75 e $150 \mathrm{~kg} \mathrm{~N} \mathrm{ha}^{-1}$, aos $8 \mathrm{DAC}$, as quais não diferiram entre si ( $\mathrm{P}>0,05$, Teste de Bonferroni), os valores de ICF aumentaram significativamente com as doses de $\mathrm{N}$ até os 16-24DAC, decaindo, não significativamente, na amostragem final. O máximo ICF $(52,1)$ foi registrado aos 16DAC, numa parcela que recebeu a maior dose de $\mathrm{N}$. Independentemente de data de amostragem, valores de ICF aumentaram, em média, 43\%, considerando-se a

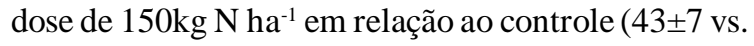
30 \pm 2 ICF, respectivamente), ou seja, a adubação teve um efeito comparativamente menor sobre o ICF do que sobre o teor de CFT. Para o conhecimento dos autores, não existe registro prévio do uso do ClorofiLOG em cultivares do gênero Cynodon spp., portanto não se dispõe de um termo de comparação direta para aferir a validade dos presentes resultados. Entretanto, como o ClorofiLOG e o SPAD utilizam a mesma escala de medição, é possível realizar algumas inferências. PREMAZZI \& MONTEIRO (2002), avaliando as respostas do Tifton-85 à aplicação de $\mathrm{N}$ no solo, em vasos mantidos em casa de vegetação, obtiveram valores máximos de leituras SPAD entre 39,4 e 44,2, dependendo do ciclo de rebrota, ou seja, de ordem similar aos aqui observados. Por outro lado, como mostra a figura $1 \mathrm{C}$, o grau de associação entre os valores médios obtidos com ambos os métodos de quantificação de clorofila correspondeu a um coeficiente $\mathrm{r}=0,721$ ( $\mathrm{P}=0,0081)$. Esse valor de correlação é de magnitude similar aos obtidos com outras espécies, em estudos que utilizaram o SPAD, sob condições de campo (SIBLEY et al., 1996; ARGENTA et al., 2001b). A 


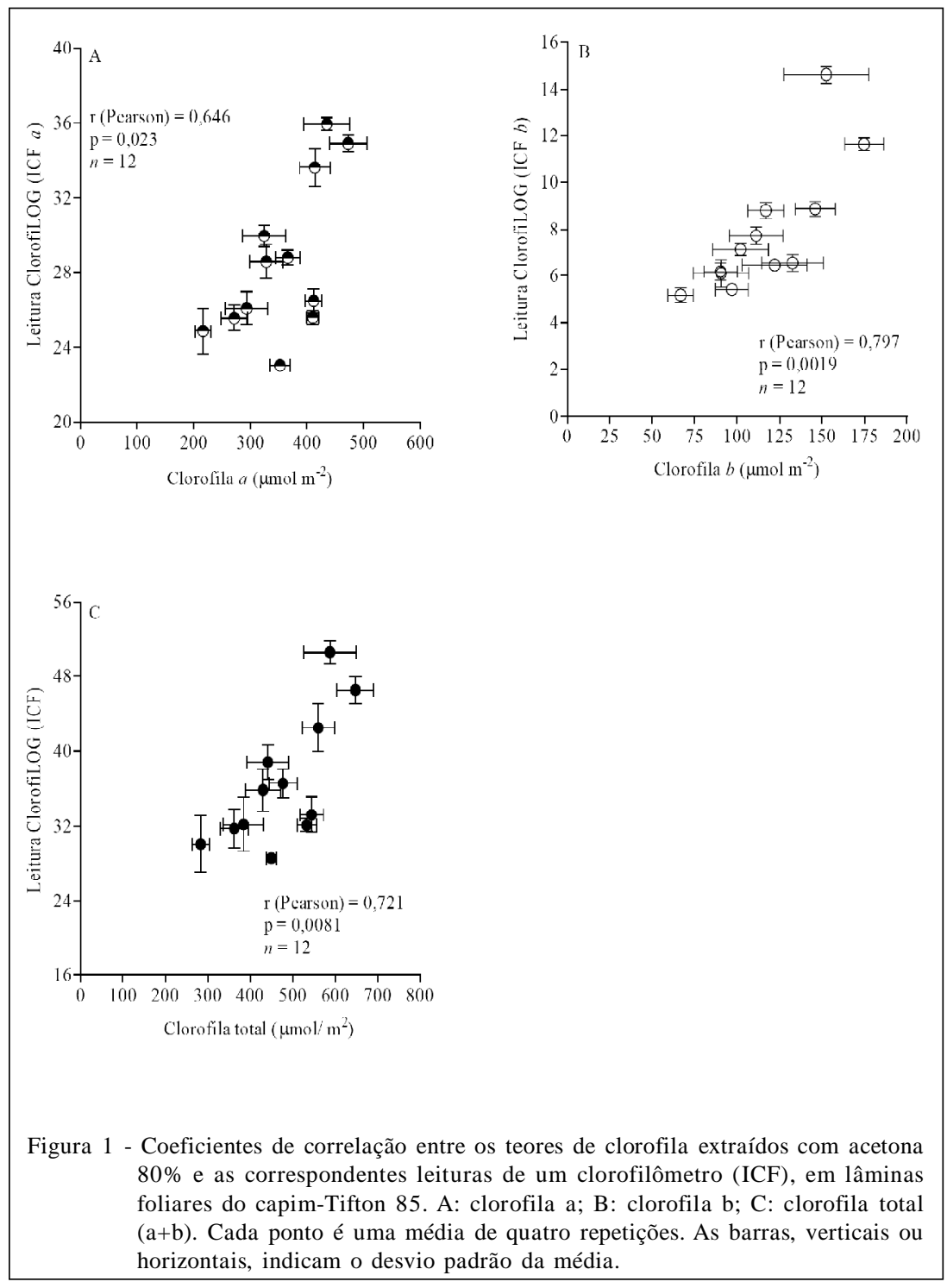

correlação entre os teores de CFT e os seus correspondentes ICF exprime a contribuição aditiva das clorofilas a e b. Com relação à clorofila $a$, os seus teores variaram entre 200 e $510 \mu \mathrm{mol} \mathrm{m} \mathrm{m}^{-2}$, e os seus correspondentes valores de ICF entre 22,8 e 36,9 $(r=0,646$, Figura 1A), enquanto que a clorofila $b$ variou entre 58 e $190 \mu \mathrm{mol} \mathrm{m}^{-2}$ correspondente a valores de ICF entre 4,9e 15,2 (r=0,797, Figura 1B).

Como mencionado previamente, houve diferenças associadas às datas de amostragem. Para a CFT, excluindo-se as médias correspondentes aos $8 \mathrm{e}$ 28DAC, o valor do coeficiente de correlação melhorou expressivamente, passando para $\mathrm{r}=0,926(\mathrm{P}=0,0086)$, indicando ser o período compreendido entre $16 \mathrm{e}$ 24DAC, o mais apropriado para a quantificação das clorofilas. A figura 2 mostra a natureza linear da relação entre ambos os métodos de determinação das clorofilas, tanto aos 16 quanto aos 24 dias (Figuras 2A e B, respectivamente).

Tomados em conjunto, os dados obtidos permitem afirmar que o ClorofiLOG constitui um instrumento adequado para a determinação indireta dos teores relativos das clorofilas a, b e total na gramínea forrageira Tifton 85. Por outro lado, à medida que seu desempenho foi sensível a variações espaciais e temporais, induzidas por disponibilidades diferenciadas de $\mathrm{N}$ no solo, este clorofilometro pôde constituir uma excelente ferramenta no manejo da adubação nitrogenada dessa forrageira, assim como de outras representantes do gênero Cynodon spp.

Ciência Rural, v.42, n.12, dez, 2012. 


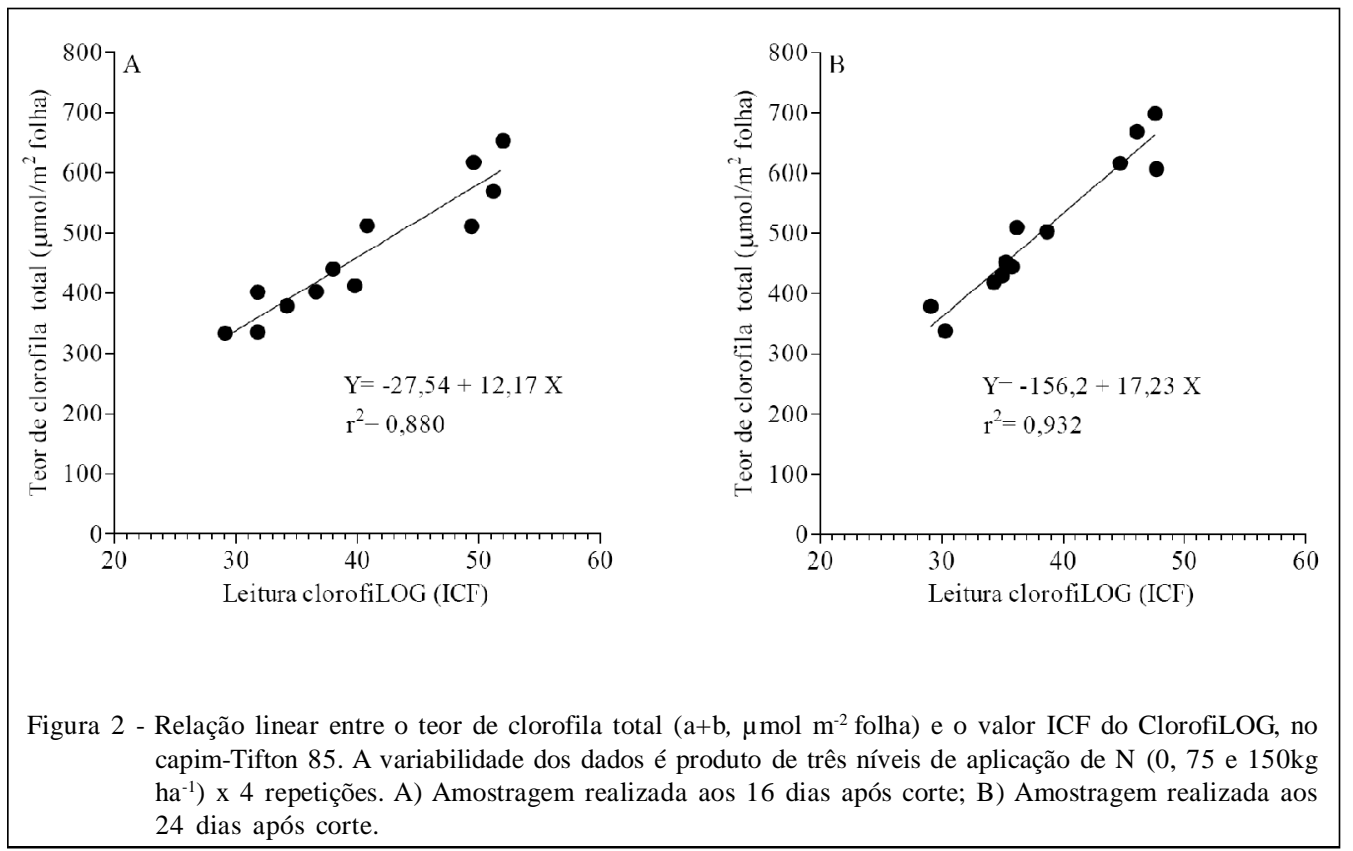

\section{REFERÊNCIAS}

ARGENTA, G. et. al. Clorofila na folha como indicador do nível de nitrogênio em cereais. Ciência Rural, v.31, n.4, p.715-722, 2001a. Disponível em: <http://www.scielo.br/ scielo.php?script=sci_arttext \& pid=S0103$84782001000400027 \& \operatorname{lng}=e n \& n r m=i s o>$. Acesso em: 28 ago. 2012. doi: $10.1590 / \mathrm{S} 0103-84782001000400027$.

ARGENTA, G. et. al. Relação da leitura do clorofilometro com os teores de clorofila extraível e de nitrogênio na folha de milho. Revista Brasileira de Fisiologia Vegetal, v.13, n.2, p.158-167, 2001b. Disponível em: <http://www.scielo.br/ scielo.php ? script $=\mathrm{sci}_{-}$art text \& pid= 0103 $31312001000200005 \& \operatorname{lng}=e n \& n r m=i s o>$. Acesso em: 28 ago. 2012. doi: 10.1590/S0103-31312001000200005.

ARNON, D.I. Copper enzymes in isolated chloroplasts. Polyphenoloxidase in Beta vulgaris. Plant Physiology, v.24, n.1, p.1-15, 1949. Disponível em: <http://www.ncbi.nlm.nih.gov/ pmc/articles/PMC437905/>. Acesso em: 28 ago. 2012.

BARBIERI JUNIOR, É. et al. Comparação de métodos diretos de extração e quantificação dos teores de clorofilas em folhas do capimTifton 85. Ciência Rural, v.40, n.3, p.633-636, 2010. Disponível em: <http://www.scielo.br/scielo.php?script=sci_arttext\&pid=S0103$84782010000300022 \& \operatorname{lng}=e n \& n r m=i s o>$. Acesso em: 28 ago. 2012. doi: $10.1590 / \mathrm{S} 0103-84782010000300022$.

FALKER, Automação agrícola. Manual do medidor eletrônico de teor clorofila (ClorofiLOG/CFL 1030). Porto
Alegre, 2008. 33p. Disponível em: <http://www.falker.com.br/ produto_download.php?id=4>. Acesso em: 28 ago. 2012.

MARKWELL, J. et. al. Calibration of the Minolta SPAD-502 leaf chlorophyll meter. Photosynthesis Research, v.46, n.3, p.467-472, 1995. Disponível em: <http://www.springerlink.com/ content/b3q41327j2387225/>. Acesso em: 28 ago. 2012. doi: 10.1007/BF00032301

MOTULSKY, H.; CHRISTOPOULOS, A. Fitting models to biological data using linear and nonlinear regression: a practical guide to curve fitting. Oxford: Oxford University, 2004. 352p.

ORTUZAR-IRAGORRI, M.A. et al. N-Tester use in soft winter wheat: evaluation of nitrogen status and grain yield prediction. Agronomy Journal, v.97, n.5, p.1380-1389, 2005. Disponível em: <https://www.soils.org/publications/aj/pdfs/97/ 5/1380>. Acesso em: 28 ago. 2012. doi: 10.2134/ agronj2004.0268.

PREMAZZI, L.M.; MONTEIRO, F.A. Produção do capimTifton 85 submetido a doses e épocas de aplicação de nitrogênio após o corte. Boletim da Indústria Animal, v.59, n.1, p.116, 2002. Disponível em: <http://www.iz.sp.gov.br/ pesq_bia.php?id=240>. Acesso em: 28 ago. 2012.

SIBLEY, J.L. et al. Foliar SPAD-502 meter values, nitrogen levels, and extractable chlorophyll for red maple selections. Hortscience, v.31, n.3, p.468-470, 1996. Disponível em: <http://hortsci.ashspublications.org/content/31/3/468.full.pdf〉. Acesso em: 28 ago. 2012. 